\title{
A PRÉ-INICIAÇÃO CIENTÍFICA: UM PROCESSO DE INCLUSÃO OU SEGREGAÇÃO?
}

\author{
Antônio José Silva-Gonçalves ${ }^{1}$, Sandra Maria Gomes de Azevedo², Jonathan \\ Goncalves-Oliveira ${ }^{3}$ e Valéria da Silva Trajano ${ }^{4}$ \\ ${ }^{1}$ Instituto Oswaldo Cruz / Fundação Oswaldo Cruz e Universidade Estácio de Sá/UNESA, Brasil - ajsg@ioc.fiocruz.br \\ 2,3,4 Instituto Oswaldo Cruz / Fundação Oswaldo Cruz Brasil \\ sandraazevedocvt@gmail.com;goncalvesjohn03@gmail.com;valeria.trajano@ioc.fiocruz.br
}

\begin{abstract}
Resumo. A iniciação científica e pesquisa é um eixo estruturante do ensino médio. Entretanto, a iniciação científica desenvolvida no ensino médio é advinda de programas de Pré-Iniciação Científica (PIC). Esses Programas são desenvolvidos em instituições de pesquisa e ensino de nível superior. A inserção de jovens nesses Programas é meritocrática e tem por base a inclusão social. Esses critérios elimina uma parcela representativa de estudantes. Este estudo analisou a opinião de 126 estudantes do ensino médio que não participaram do Programa PIC, "Projeto Jovens Talentos para Ciência (FAPERJ)" (PJT), do Colégio Estadual Deodato Linhares, na cidade de Miracema, Rio de Janeiro, Brasil. A metodologia foi exploratória e qualitativa, cuja questão inicial foi: Para você o que é o PJT? Os depoimentos foram analisados pela Técnica de Análise de Conteúdo, onde $55 \%$ dos participantes eram do gênero feminino, $40 \%$ do gênero masculino e $5 \%$ não se classificaram nos dois gêneros, dentre eles, $27 \%$ caracterizaram o Programa como um PIC; 7,9\% declararam desconhecer o Programa; $14,3 \%$ menosprezaram o Programa e 43,6\% consideraram o Programa como uma oportunidade de crescimento intelectual, social ou profissional. Esses resultados apontaram falhas de divulgação, desenvolvimento do processo de segregação escolar e a necessidade de discussão sobre o conceito de inclusão social dos Programas PIC instituidos no país.
\end{abstract}

Palavras-chave: Inserção; Educação Básica; Projetos Educacionais; Inclusão; Segregação Escolar

\section{THE SCIENTIFIC PRE-INITIATION: AN INCLUSION OR SEGREGATION PROCESS?}

\begin{abstract}
Scientific Initiation and Research is a structuring axis of high school. However, the scientific initiation developed in high school comes from Scientific Pre-initiation Programs (PIC). These Programs are developed in higher education research and teaching institutions. The inclusion of young people in these Programs is meritocratic and is based on social inclusion. These criteria eliminate a representative portion of students. This study analyzed the opinion of 126 high school students who did not participate in the PIC Program, "Project Young Talents for Science (FAPERJ)" (PJT), from Colégio Estadual Deodato Linhares, in the city of Miracema, Rio de Janeiro, Brazil. The methodology was exploratory and qualitative, whose starting question was: For you what is PJT? The testimonies were analyzed by the Content Analysis Technique, where $55 \%$ of the participants were female gender, $40 \%$ of male gender and $5 \%$ no binaries gender, among them, $27 \%$ characterized the Program as a PIC; $7.9 \%$ declared they were unaware of the Program $14.3 \%$ dismissed the Program and $43.6 \%$ considered the Program as an opportunity for intellectual, social or professional growth. These results pointed out the lack of disclosure, the development of the school segregation process and the need to discuss the concept of social inclusion of the PIC Programs instituted in the country.
\end{abstract}

Keywords: Insertion; Basic Education; Educational Projects; Inclusion; School Segregation

\section{INTRODUÇÃO}

O ensino médio brasileiro faz parte da educação básica e integra uma Base Curricular Comum com outra diversificada que compreende a formação para o trabalho, de caráter 
técnico; iniciação científica e tecnológica; ampliação e formação cultural. A iniciação científica e pesquisa são campos estruturantes do ensino médio brasileiro, última etapa da educação básica (Brasil, 2009). Nessa fase, os estudantes devem ter experiências de produção e socialização de conhecimentos científicos e tecnológicos que ocorrem na sociedade relacionando teoria com a prática. Apesar da iniciação científica e pesquisa ser um dos campos estruturantes do ensino médio brasileiro, ainda é um campo de atuação negligenciado.

A iniciação científica desenvolvida no ensino médio é proveniente de programas de PréIniciação Científica (PIC). O primeiro PIC, no Brasil, foi fruto do sonho de um pesquisador que por base em suas experiências passadas acreditou na formação do jovem pesquisador, na tenra idade. Com base nesse sonho nasceu o Programa de Vocação Científica (PROVOC), em 1986, na Escola Politécnica de Saúde Joaquim Venâncio, uma unidade técnico-científica da Fundação Oswaldo Cruz/Ministério da Saúde, localizada na cidade do Rio de Janeiro, Brasil (Medeiro et al., 2016).

Desde então outros PIC surgiram no Brasil, utilizando o Provoc como modelo. Esses PIC são instituídos por política pública educacional, financiados pelo Estado e em parcerias com universidades, institutos pesquisa e tecnologia. O modelo pedagógico desenvolvido é baseado em metodologias ativas, conhecido pelo formato de projetos, sob a orientação de pesquisadores qualificados com mestrado ou doutorado. Além disso, os PIC são direcionados para estudantes da rede pública, algumas exceções permitem a participação de estudantes da rede privada. Esses PIC têm como um dos objetivos a inclusão de jovens de classes sociais desfavorecidas (Arantes \& Peres, 2015).

A importância das práticas de PIC na educação básica tem sido repensada e valorizada em diferentes instituições, além de ser alvo de estudo de pesquisadores que registram e analisam as experiências com jovens participantes de tais Programas. Esses pesquisadores são unânimes em concluir que a participação de estudantes de educação básica em atividades de pesquisa é positiva tanto os estudantes envolvidos quanto os pesquisadores que necessitam de integrantes para o desenvolvimento dos projetos, e incentivando esses estudantes a seguirem uma carreira acadêmica (Sousa \& Filipecki, 2009; Sousa, 2010; Conceição, 2012; Cardoso, 2016).

Devido ao apoio de agências de fomento para essa atividade diminui o fosso entre ensino médio e ensino superior, além de contribuir para a propagação da pesquisa e da iniciação 
científica no ensino médio. Por outro lado, há uma parcela de estudantes não é selecionada, sendo excluída do programa. Neste estudo analisamos a opinião de estudantes que não participaram do Programa de PIC, denominado "Projeto Jovens Talentos para Ciência (FAPERJ)", desenvolvido no Colégio Deodato Linhares, na cidade de Miracema no noroeste do estado do Rio de Janeiro, Brasil.

\subsection{Projeto Jovens Talentos para Ciência (FAPERJ)}

O "Projeto Jovens Talentos para Ciência (FAPERJ)" faz parte de um Programa desenvolvido pelas instituições: Fundações Centro de Ciências do Estado do Rio de Janeiro (CECIERJ) e a Fundação de Amparo à Pesquisa Carlos Chagas Filho (FAPERJ). O Programa tem parcerias com Instituições de Pesquisa e Universidades Públicas e Privadas do Estado do Rio de Janeiro, abraçando diferentes áreas do conhecimento. Por meio desse Programa, estudantes da rede pública estadual de ensino médio e profissional são inseridos na PIC. Os objetivos do Programa são (i) selecionar estudantes com interesse pela ciência e potencial para atuar em pesquisa científica; (ii) estimular a formação dos estudantes; (iii) identificar novos talentos para atuação profissional no campo do saber científico; (iv) contribuir para a difusão dos conhecimentos científicos, desmitificando a ciência e articulando pesquisa e ensino.

O ingresso de jovens no Programa é seletivo e os critérios de inclusão são: (i) média igual ou superior a sete; (ii) não apresentar dependência em disciplinas curriculares; (iv) faixa etária de 15 a 18 anos (v) entrevista oral e escrita. Na entrevista, o estudante é avaliado quanto à disponibilidade de carga horária, interesse por atividades científicas e facilidade de argumentação.Todos os estudantes que não se enquadram em algum desses critérios são excluídos.

O Programa compreende dois estágios. Estágio Inicial, que tem duração de seis meses (julho-dezembro). No final do estágio, o estudante apresenta um relatório das atividades realizadas e poderá solicitar a renovação do estágio para o estágio avançado que é direcionado aos jovens que demonstraram mais afinidade com a atividade científica e desejam dar continuidade aos seus projetos de pesquisa por mais doze meses. O estudante finaliza com a apresentação do relatório final seus resultados na Jornada Científica, que é realizada anualmente pela coordenação do projeto, em cooperação com a FAPERJ e com as instituições parceiras. A partir de 18 de julho de 2003, os programas passaram a contar com o apoio do Conselho Nacional de Desenvolvimento Científico e Tecnológico/MCT 
(CNPq), que disponibiliza bolsas de estudo de PIC júnior, as bolsas têm como finalidade auxiliar no deslocamento e alimentação dos participantes. Atualmente, o valor da bolsa é de $\mathrm{R} \$ 210,00$ (duzentos e dez reais). Vale ressaltar que no Brasil, em 26 de dezembro de 2019 o salário mínimo nacional era de $\mathrm{R} \$ 998,00$ (novecentos e noventa e oito reais). Portanto esses estudantes recebem $21,04 \%$ desse salário, que representa em dólares americanos, nessa época, $\bigcup \$ 50,60$ (cinquenta dólares e sessenta cents) ou $€ 46,26$ (quarenta e seis euros e vinte e seis cents).

\subsection{Projetos Jovens Talentos para Ciência (FAPERJ) na cidade de Miracema}

No ano de 2010 foi implantado o Projeto Jovens Talentos para Ciência (FAPERJ), no Colégio Estadual Deodato Linhares (CEDL), na cidade de Miracema no Noroeste do estado do Rio de Janeiro, com a participação de quatro estudantes do segundo ano do ensino médio. Esses estudantes foram selecionados segundo os critérios estabelecidos no edital da FAPERJ, descrito anteriormente. Num estudo retrospectivo sobre esse Programa analisouse o período de vigência de 2010 a 2017 no qual 137 jovens participaram, dentre eles, 55\% eram do gênero feminino e $45 \%$ do gênero masculino (Azevedo, 2019). Do total de participantes, 94\% ingressaram no nível superior, e nesse grupo de graduandos, 64\% cursaram universidades públicas e $56 \%$ cursaram universidades privadas com bolsas de $100 \%$ pelo Programa Universidade para todos (PROUNI). Vale ressaltar que 30\% optaram pela área de Ciências da Saúde e 19\% pelas Ciências Biológicas. Durante esse período, foram desenvolvidos 93 projetos, sendo que 32, ainda estão em execução. Os projetos abordavam a realidade da cidade, seja histórica, literária, social, ambiental e/ou de saúde, atualmente, participam do Programa 14 estudantes desenvolvendo 20 projetos.

O CEDL tem, em média, mil estudantes por ano, mas seu espaço físico pode comportar até dois mil estudantes. Considerando essa informação, o número de vagas para participar no Programa é limitado e com um quantitativo de vagas baixo em relação a quantidade de estudantes na escola.

Vale ressaltar que o Programa em Miracema tem um dos maiores quantitativos de vagas em todo estado do Rio de Janeiro, em média recebe 20 bolsas anualmente. Dessa forma, um grande quantitativo de estudantes fica impossibilitado de participar, mesmo atendendo aos critérios. Portanto, o processo seletivo e de concessão das bolsas é bem criterioso e meritocrático. Muito embora, as premissas do PIC partem de um princípio de inclusão de jovens de classes sociais mais desfavorecidas ao meio científico. O PIC tem como 
característica ser desenvolvido no interior de uma instituição de pesquisa e ensino, como universidades ou centros de pesquisas. O PIC de Miracema é uma exceção, pois estava vinculado a um Centro de Vocação Tecnológica (CVT) de Miracema, porém suas atividades foram encerradas. Dessa forma, o PIC permaneceu na cidade utilizando as dependências do CEDL.

A lógica de inclusão dos participantes no PIC é ambígua, pois tanto é meritocrática quanto tenta compensar as desigualdades sociais, uma vez que tenta favorecer jovens de classes menos favorecidas. Apesar das premissas do PIC, ao selecionar seus integrantes, não considera que todos os estudantes devam desenvolver os seus talentos independente do seu desempenho escolar, de suas condições físicas e financeiras. Fato compreensível, pois esses PIC não foram criados no intuito de desempenhar o papel da escola, pois esta por muitos anos têm sido mais excludente do que inclusiva, na maioria dos países, às vezes, de forma sutil (Resende, 2003; Dubet, 2004; Dayrell \& Jesus, 2016). Essa meritocracia que rege os PIC tem sido questionada, a ponto de determinados PIC inserir estudantes em seus Programas independente da classe social desses estudantes (Ferreira, 2003).

Além disso, devemos considerar que esse processo de inclusão meritocrático pode levar a segregação, principalmente quando o Programa é desenvolvido dentro de uma instituição escolar. O termo segregação está relacionado a um tratamento desigual, na maioria das vezes injusto, sendo atribuído a uma pessoa ou a um grupo baseado em preconceitos de diferentes ordens, como étnicos, religiosos, sexual, de gênero, dentre outros. A segregação escolar ocorre quando há agrupamentos de indivíduos por características comuns que recebem tratamento diferenciado. Esse agrupamento ou segregação na educação, tem sido abordado principalmente quando estão relacionados à associação de dimensões territoriais e de locais de residência. Exemplo disso são estudos que relatam os processos de alistamento escolar em diferentes cidades do Brasil ou do mundo, que favorecem a segregação social e urbana (Costa \& Bartholo, 2014; Mendes, 2017). Geralmente a segregação afeta diretamente a qualidade e a equidade do sistema educacional acarretando a injustiça social.

A segregação de estudantes por condições específicas pode influenciar na qualidade do ensino e no desenvolvimento intelectual desses estudantes, que, por conseguinte são tratados no meio escolar de forma diferenciada. Vygotsky (1998) desde o século XX defendia a interação social e por meio de seus estudos, demonstrou que o processo ensino e aprendizagem são enriquecidos entre indivíduos de diferentes faixas etárias, assim como 
a interferência do meio cultural nesses processos. Características comuns, principalmente de desigualdade social, cultural, econômica, até mesmo a segregação de estudantes violentos geram a evasão escolar, pois estes estudantes que apresentam baixo desempenho escolar ou tem dificuldade de construção de novos conhecimentos podem se tornar estagnados e desmotivados (Haahr et al., 2005; Brito \& Costa, 2010; Bartholo \& Costa, 2014).

Vários artigos relatam a experiência de participantes de PIC (Sousa \& Filipecki, 2009; Sousa, 2010), mas há escassez de informações a respeito dos estudantes que participaram do processo seletivo e não foram selecionados e também dos que não puderam participar por não apresentar os critérios mínimos do processo seletivo. Neste estudo analisamos as opiniões de 126 estudantes do Colégio Estadual Deodato Linhares que não participaram do Programa. Este estudo foi uma iniciativa de Jovens Talentos do ano de 2019, que tiveram a curiosidade de saber a opinião dos estudantes que não participaram do Programa.

\section{METODOLOGIA}

Este estudo apresenta um caráter exploratório, com uma abordagem qualitativa, quando o conhecimento é considerado um processo socialmente construído pelos indivíduos numa interação constante, dentro de uma determinada realidade. Uma grande quantidade de informações detalhadas dessa realidade nos possibilita compreender a sua totalidade, e pode levar à resolução de problemas (André, 2005). Portanto, para pesquisar essa realidade, precisamos estar imersos nela ou no mínimo mais próximos para conhecer os códigos da comunidade, como a cultura, a linguagem, as vivências, entre outros (André, 2005). Estar imerso, não significa que o pesquisador vai interferir com sua visão de mundo, seus valores e emoções na análise dessa realidade. Muito embora nenhuma pesquisa qualitativa ou quantitativa seja neutra (Minayo, 1998).

O presente estudo foi desenvolvido em 2019 e partiu da curiosidade de estudantes do PIC Jovens Talentos para Ciência (FAPERJ), desenvolvido no Colégio Estadual Deodato Linhares (CEDL) localizado na cidade de Miracema, no noroeste do estado do Rio de Janeiro/Brasil. A questão norteadora deste estudo foi: Como os estudantes do CEDL que não participaram do Programa Jovens Talentos para Ciência (FAPERJ) o percebem?

Esta pesquisa teve como objetivo avaliar as percepções desses estudantes sobre o Programa Jovens Talentos para Ciência (FAPERJ). Para tanto, os Jovens Talentos (JT) construíram um questionário como instrumento de coleta de dados, com seis questões 
abertas e validadas pelo Comitê de Ética da Fundação Oswaldo Cruz, sob o número de registro: 2.109.868. No entanto, neste estudo analisaremos apenas uma questão - Para você o que é o Projeto Jovens Talentos? - devido à riqueza dos achados e o espaço limitados para a exposição dos dados e das discussões e reflexões geradas.

O questionário é uma técnica de coleta de dados utilizada para obter informações sobre um tema específico. Essa técnica é muito utilizada em pesquisas qualitativas porque permite questionar um elevado número de indivíduos em curto espaço de tempo. As perguntas formuladas devem ser em linguagem clara e objetiva e o questionário deve ser testado quanto a sua pertinência (Gil, 1987).

Os resultados preliminares nos levaram a refletir sobre os PIC e suas problemáticas. Além disso, os achados expostos neste estudo podem servir como um passo inicial para outras pesquisas sobre o assunto de forma mais profunda. Os depoimentos dos questionários foram analisados segundo a Técnica de Análise de Conteúdo de Bardin (2011), que se apropia de um conjunto de técnicas de análises das comunicações, no intuito descrever o conteúdo das mensagens ao mesmo tempo nos permite a inferência de conhecimentos relacionados á produção e recepção de mensagens. Nesse tipo de análise de conteúdo, podemos nos cercar de técnicas precisas e objetivas que nos permite a descoberta do verdadeiro significado da mensagem, ou seja, o que está por trás do texto/imagem ou discurso (Bardin, 2011).

Bardin (2011) divide a análise de conteúdo em três fases distintas que compreende inicialmente (i) pré-análise; fase na qual realizamos uma leitura flutuante dos depoimentos do questionário, constrindo nossas primeiras impressões sobre eles; Nessa fase também observamos as seguintes regras: (a) a regra da exaustividade, todos os elementos contidos nos dados são analisados exaustivamente; (b) a regra da representatividade, na qual o quantitativo da amostra deve ser representativo do universo pesquisado; (c) regra da homogeneidade, os documentos analisados devem abordar o mesmo assunto; (d) regra da pertinência, os documentos analisados devem atender aos objetivos da pesquisa. Na fase de (ii) exploração do material consistiu na exploração do material, nessa fase se construímos as unidades de registro, de contexto e classificação por analogias. Nessa etapa analisamos os discursos para criar as categorias, logo frases como Um projeto que ajuda os alunos a ter mais contato com a ciência em si foram agrupadas com outras de mesmo contexto tais como: um projeto de iniciação científico onde ele insere o jovem no meio científico e diminui o analfabetismo cientifico introduzindo projetos ou um projeto de 
pesquisa que envolve várias áreas da ciência. Dessa forma na etapa de (iii) tratamento dos resultados provenientes da análise, com esses depoimentos constituímos os núcleos de sentido que compõem a comunicação e cuja presença ou frequência de aparição pode significar alguma coisa para o objetivo analítico. Nesse caso o núcleo de sentido foi : projeto de iniciação científica. Esses dados condensados e as informações destacadas para análise, possibilitou as interpretações inferenciais, passando por uma análise intuitiva, reflexiva e crítica, como destaca Bardin (2011). Estas duas etapas foram realizadas para a identificação de todos os núcleos de sentidos apresentados nesse artigo, assim como o seu percentual nos 126 depoimentos analisados. Ressaltamos que a análise de conteúdo pode ser também uma análise de significados, pois ela pode ser aplicada a diversos discursos com uma descrição objetiva, sistemática e quantitativa quanto ao conteúdo, extraindo seus significados. Ressaltamos que a discussão deste artigo se apoia nos referenciais temáticos do campo de estudo de Programas PIC, exclusão e inclusão social e escolar, com discussões e reflexões sobre os pontos convergentes e divergentes e apresentados na discussão dos resultados desta pesquisa.

\section{RESULTADOS/DISCUSSÃO}

Os sujeitos da pesquisa foram 126 estudantes do ensino médio do CEDL, em Miracema, cidade localizada no noroeste do estado do Rio de Janeiro/Brasil. O perfil desse grupo se caracteriza, em sua maioria, por estudantes do sexo feminino. Esse perfil vai ao encontro dos dados do Instituto Nacional de Estudos e Pesquisa Educacionais Anísio Teixeira (INEP), que demonstra que cresce o número de mulheres em todos os níveis de ensino, no Brasil e esse crescimento se consolida como maioria a partir do ensino médio (INEP, 2019). Apesar desse número crescente, o assunto gera polêmica sobre a valorização da mulher no mundo do trabalho, nas escolhas profissionais e as implicações com questões de gênero, dentre outras (Barros \& Mourão, 2018).

Quando questionados sobre o que é o Projeto Jovens Talentos, 27\% (34/126) dos participantes caracterizaram como um Programa de PIC, demonstrando conhecer o Programa. "O projeto jovens talentos é um projeto de iniciação científica onde ele insere o jovem no meio científico e diminui o analfabetismo cientifico introduzindo projetos." e "É um projeto de pesquisa que envolve várias áreas da ciência." O programa Jovens Talentos para Ciência (FAPERJ) existe nessa unidade escolar desde 2010, portanto esperávamos que um grupo bem maior de estudantes conhecesse os objetivos do projeto. Nesses dados, identificamos uma falha no processo de divulgação interna do Programa, pois 7,9\% (10/126) 
declararam desconhecer o Programa, 3,9\% (5/126) que responderam de forma evasiva, como: interessante, projeto da escola, os 2,4\% (3/126), não responderam a questão, esse percentual resulta em $14,2 \%$ de desconhecimento total ou parcial sobre o programa.

Esses relatos nos levaram a questionar o processo de divulgação interna do Programa no interior da unidade escolar. Ressaltamos que o Programa possui uma página no Facebook criada pelos participantes do Programa JT, desde 2017. Além disso, verificamos que $14,3 \%$ (18/126) se referiram ao Programa com menosprezo. "jovens que... trabalham com ciências e usam jaleco fora do ambiente adequado?”, “The Littes lab coats...”, “Uma perda de tempo, pois em nada acrescenta". Dentre os $14,3 \%$ há aqueles que até gostam do projeto, mas menospreza mesmo assim. "Um projeto legal, mas que eu não tenho interesse de entrar, porque parece enjoativo, chato e muito ranço (algumas pessoas legais), mas acho que são pessoas normais, devem ser tratadas iguais." e "É um projeto legal, porém atrapalha o desempenho de alguns alunos."

Uma percentual minotário porém significativo, desconhece e menospreza o programa, sendo mais de um quarto do total de participantes avaliados $(28,5 \%)$.

Esses estudantes adotam atitudes de ressentimento típicas de adolescentes, que se sentem injustiçados ou excluídos, de um determinado grupo social. Por outro lado, nesse achado podemos perceber a construção de um processo de segregação se instalando no meio escolar. Um grupo de estudantes que se acham preteridos, portanto menosprezam o Programa e formam opiniões de valores que evidenciam a desigualdade escolar.

Ao mesmo tempo, podemos ter outro grupo que se acha superior em relação ao anterior.

Esses resultados nos levaram a refletir sobre o processo de segregação que está se instalando no ambiente escolar, gerado pelo Programa. A segregação escolar advinda dos padrões de distribuição de oportunidades escolares é um tema discutido desde meados do século XX na América do Norte (Costa \& Bartholo, 2014), para o qual as propostas de correção são escassas, mas que geram reflexões sobre a importância da igualdade de oportunidade para todos no ambiente escolar. Muito embora, não tenhamos como solucionar de imediato o problema, reconhecemos essa realidade e a necessidade de aprofundar os nossos estudos em relação a essa temática, na busca de mecanismos para enfrentamento dessa problemática gerada nesse contexto escolar. 
Neste estudo identificamos também que 43,6\% (55/126) dos estudantes consideraram o Programa como uma oportunidade de crescimento intelectual, social ou profissional, que ajuda os estudantes a terem acesso a estudos mais elaborados. "É um projeto que tem um ensino mais elevado para alunos que tem potencial de ter um futuro bem sucedido em relação ao emprego." Outro achado significativo que nos alertou, foi a aceitação da exclusão de uma parcela dos estudantes $(44,5 \%)$, isso é um sinal que também requer questionamentos. Esse grupo considera normal não estar entre os melhores e aceitar um ensino diferenciado sem questionar o papel da escola, como reprodutora de desigualdades sociais, demonstrando a falta de criticidade desses estudantes, uma das características primordiais para o enfrentamento das condições adversas da sociedade brasileira (Resende, 2003).

A exclusão social é um fator muito marcante na sociedade brasileira, principalmente, as exclusões determinadas por fatores socioculturais e os jovens são parte integrante desse processo, no que tange a educação. A cada ano, vários jovens abandonam as escolas ou consideram que concluíram seus estudos quando terminam a educação básica, muitos nem sequer têm ideia de que existem outras aspirações acadêmicas. Esse pensamento é compatível com a escola republicana dos anos 30 do século $\mathrm{XX}$, na França, quando a maioria dos estudantes de classes menos favorecidas apenas concluía a escolaridade obrigatória. Já a partir da década de 60 , ficou evidente o papel excludente da escola e sua reprodução das desigualdades sociais, os estudantes mais fracos são impostos a uma subvalorização da sua mão de obra devido a baixa escolarização (Resende, 2003; Dubet, 2004; Dayrell \& Jesus, 2016).

No discurso dos estudantes nos deparamos com uma das falhas do PIC, que reproduz à escola que seleciona os estudantes pelo seu desempenho, os mais fracos são excluídos do acesso a uma carreira acadêmica como podemos constatar em um dos discursos: "Encontrar jovens bons e ajuda-los em seu desempenho." Os métodos pedagógicos ativos requerem uma condição social melhor dos estudantes e pais que se mobilizem e estimulem seus filhos, uma forma sutil de seleção. Vários problemas se intercruzam com os expostos, na tentativa de contornar esse papel social da escola como as condições socioeconômicas, mas ao analisar o conjunto, nos deparamos com o afastamento da escola do seu papel primordial de preparar o indivíduo para a sociedade em que vivem e para a transformação de sua realidade, por mais desigual que seja (Dayrell \& Jesus, 2016). 
Os programas de pré-iniciação científica no Brasil, tem demonstrado resultados surpreendentes, que podem ser constatados por diversos relatos de participantes dos programas por meio de transformações de vida e aspirações. A metodologia de projetos científicos já estimulou vários jovens de classe menos favorecidas a seguirem a vida acadêmica e se realizarem profissionalmente (Azevedo, 2019). No entanto, temos que reconhecer que o modelo não pode fortalecer um sistema arcaico centrado na meritocracia, "onde alguns alunos tem a oportunidade de pesquisar mais e se preparam para a faculdade", como declarou um dos estudantes. O programa deveria se expandir de modo a alcançar todos os jovens e proporcionar uma escola de qualidade e igualitária que forma cidadãos críticos e aptos para a realidade do século XXI.

Para tanto, com a inserção da iniciação científica e pesquisa como eixo estruturante do ensino médio, que está em fase de transição para ensino integral, os PIC poderiam adotar ou se instalar em uma escola pública ou privada, pois não defendemos a exclusão, possibilitando a participação de todos os educandos. Essa inicitiva nos possibilitaria constar que a metodologia de projetos, desenvolvida por esses programas é capaz de transformar e incentivar, até mesmo estudantes de baixo rendimento escolar, que podem apresentar um grande potencial criativo. Vários autores têm demonstrado que o sucesso desses programas, na maioria das vezes, está centrado na metodologia ativa de projetos. Então, por que excluir? Ou Por que segregar?

\section{CONCLUSÕES}

Os programas de PIC têm incluido vários jovens de classes sociais desfavorecidas nas instituições científicas brasileiras, desde a sua criação em 1986. Pesquisas apontam a influência positiva desses programas na visão de mundo desses estudantes e o seu incentivo na vida acadêmica desses jovens. A contribuição do PIC para o desenvolvimento acadêmico, pessoal e intelectual é notório para o estudante. Dessa forma, esses jovens não só construíram conhecimentos como também transformaram a sua realidade. Contudo, nesta pesquisa identificamos pontos que alertam para um processo de segregação por um quarto dos participantes, e aproximadamente $50 \%$ dos estudantes avaliados sentem-se excluídos pelo não pertencimento ou não merecimento. Por isso, é muito cruel para essa parcela de estudantes que não tiveram a oportunidade de participar do programa, devido a não preencherem os critérios estabelecidos ou pelo número reduzido de vagas. Esses estudantes que não preencheram os critérios são na verdade, estudantes que deveriam participar desse programa também, para desenvolver habilidades e competências para a 
construção uma realidade melhor. Tanto a exclusão, como a segregação e a meritocracia no ambiente escolar, são temas de discussão e são gerados e ampliados por diversos fatores socioeconômicos e culturais. Nesta pesquisa, evidenciamos um processo de segregação escolar oriundo do processo seletivo meritocrático que faz parte da maioria dos PIC, no Brasil. Entretanto, estes programas têm sido extremamente positivos para os estudantes que participam. Por outro lado há uma escassez ou ausência de literatura sobre o quanto a exclusão de participação em um Programa como esse pode interferir na autoestima, continuidade nos estudos e a na vida profissional de estudantes não selecionados. Ressaltamos que reconhecemos o valor desses programas e acreditamos que as escolas necessitam se apropriar do eixo estruturante de iniciação científica e o desenvolver na escola, em parceria com as instituições científicas da região, com a participação de todos os estudantes de forma inclusiva e igualitária. Almejamos que este estudo exploratório possa gerar futuras pesquisas, que discutam o processo de inclusão, e políticas públicas destinadas para estudantes não selecionados para programas, para que não se sintam excluídos na comunidade escolar e não fomentem um sentimento de segregação.

Agradecimentos. (FAPERJ) pela implantação e manutenção do "Projeto Jovens talentos para Ciência (FAPERJ)", na cidade de Miracema/ Rio de Janeiro/Brasil.

\section{REFERÊNCIAS}

André, M. E. D. A. (2005). Estudo de caso em pesquisa e avaliação educacional. Brasília: Liberlivro.

Arantes, S. F., \& Peres, S. O. (2015). Programas de iniciação científica para o ensino médio no Brasil: educação científica e inclusão social. Pesquisas e Práticas Psicossociais, 10(1), 37-54. Recuperado em 24 de abril de 2020, de http://pepsic.bvsalud.org/scielo.php?script=sci_arttext\&pid=S1809$89082015000100004 \&$ lng=pt\&tlng=pt.

Azevedo S.M.G. (2019) Estudo das contribuições educacionais e sociais do Programa Jovens Talentos para Ciência Faperj, em Miracema-RJ. (Tese de Doutorado não publicada), Programa de Pós-graduação do Ensino de BiocFIOCRUZ-RJ, Rio de Janeiro-RJ.

Bardin, L. (2011). Análise de conteúdo. São Paulo: Edições 70.

Barros, S. C. V., \& Mourão, L. (2018). Panorama da participação feminina na educação superior, no mercado de trabalho e na sociedade. Psicologia \& Sociedade, 30, e174090.

Bartholo, T. L., \& Costa, M. (2014). Turnos e segregação escolar: discutindo as desigualdades escolares. Cadernos de Pesquisa. São Paulo, 44(153), 670-892.

Brasil (2009). Portaria no 971 de 09 de outubro, 2009. (2009, 13 de outubro). Institui o Programa Ensino Médio Inovador. Diário Oficial da União, Brasília, Seção 1, p. 52.

Brito, M. S. T., \& Costa, M. (2010). Práticas e percepções docentes e suas relações com o prestígio e clima escolar das escolas públicas do município do Rio de Janeiro. Revista Brasileira de Educação, 15(45), 500510 . 
Cardoso, F.S. (2016). Rede de interações como possibilidade para o desenvolvimento de pessoas com altas habilidades e vocações na área de biotecnologia. (Tese de Doutorado não publicada), Rio de Janeiro: UFF.

Conceição, A. J. (2012). Contribuições do Programa de Iniciação Científica Junior na Universidade de Londrina UEL: a formação de um habitus adequado ao campo. (Dissertação de Mestrado), Universidade Estadual de Maringá, Maringá PR.

Costa, M., \& Bartholo, T. L. (2014). Padrões de segregação escolar no Brasil: um estudo comparativo entre capitais do país. Educ. Soc., 35 (129), 1183-1203.

Dayrell, J.T., \& Jesus, R. E. (2016). Juventude, ensino médio e os processos de exclusão escolar. Educ. Soc., Campinas, 37 (135), p.407-423.

Ferreira, C. A. (2003). Concepções da iniciação científica no ensino médio: uma proposta de pesquisa. Trabalho, Educação e Saúde, 1(1), 115-130.

Gil, A. C. (1987). Métodos e Técnicas de Pesquisa Social. São Paulo: Atlas.

Haahr, J. H. et al. (2005). Explaining student performance: evidence from the international PISA, TIMSS and PIRLS surveys. Danish Technological Institute. Disponível em http://www.danishtechnology.dk. Acesso em: 28/02/2020.

Medeiros, C. M. B., Braga, C. N., Frutuoso, T. M., \& Filipecki, A.T. P. (2016). Olhares, escritos e memórias: 30 anos do Programa de Vocação Científica. 454p. Rio de Janeiro: EPSJV.

Mendes, I. A. A. (2017). Território e Segregação Escolar: um estudo da cidade de Belo Horizonte. (Tese de doutorado não publicada), Faculdade de Educação, da Universidade Federal de Minas Gerais, Brasil.

Minayo, M.C.S. (1998). A análise de conteúdo como forma de tratamento dos dados numa pesquisa qualitativa em Educação Física escolar. Movimentos. Porto Alegre, v. 16, n. 03, p. 31-49.

Dubet, (2004). O que é uma escola justa? Cadernos de Pesquisa, 34(123), 539-555.

Rezende, N. L. (2003). A escola e a exclusão. Cadernos de Pesquisa, 119, 29-45.

Sousa, I. C. F. (2010). Os egressos do Programa de Vocação Científica do Rio de Janeiro e suas concepções sobre trabalho. Ciência em Tela, 3, 1-9.

Sousa, I. C. F., \& Filipecki, A. T. P. (2009). Mentoring the relationship that makes the difference in scientific research training for youth. IEEE Professional communication society.Newsletter, USA, v.53, no7.

Vygotsky, L. S. (1988). Pensamento e linguagem. (2ª ed). São Paulo: Martins Fontes. 